\title{
LOW-FRICTION ARTHROPLASTY FOR HEALED SEPTIC AND TUBERCULOUS ARTHRITIS
}

\author{
KEVIN HARDINGE, JOHN CLEARY, JOHN CHARNLEY \\ From the Centre for Hip Surgery, Wrightington Hospital, Wigan
}

Forty hips, which had previously been the site of tuberculous or pyogenic arthritis and which had later developed a degenerative arthritis, were treated by low-friction arthroplasty some forty years after the original infection. The results suggest that, when healing of the primary infection has been followed by a long period of quiescence with acceptable function before the onset of degenerative change, the arthroplasty can be confidently expected to result in greatly improved function and that this improvement is long-lasting.

Among those patients presenting with pain and stiffness of the hips due to degenerative arthritis are a small number where there is a definite history of either septic or tuberculous arthritis in childhood or adolescence. They have been effectively cured by various forms of treatment and have usually enjoyed good function for many years, until experiencing deterioration due to the onset of degenerative change. In the past, total hip replacement has not been offered to these patients because of the hazard of stimulating a recrudescence of the previous infection.

The experience at Wrightington Hospital indicates that total hip replacement is safe in certain circumstances for these patients; and the follow-up shows that worthwhile improvement in function is maintained.
In the period July 1970 to June 1976, thirty-four patients (twenty-one women, thirteen men) with a well-defined history of old tuberculous or pyogenic infection underwent low-friction arthroplasty. Six patients had bilateral operations, making a total of forty hips. One patient died from a massive pulmonary embolus nine days after arthroplasty so that altogether thirty-nine hips were available for follow-up.

The hip infection had been tuberculous in twentyone cases, septic or pyogenic in thirteen. The age of onset varied from one year to twenty years, the average age being ten. The age of patients at the time of low-friction arthroplasty ranged from thirty-four to seventy-five years, the average age being fifty.

The function of hips in the quiescent period between

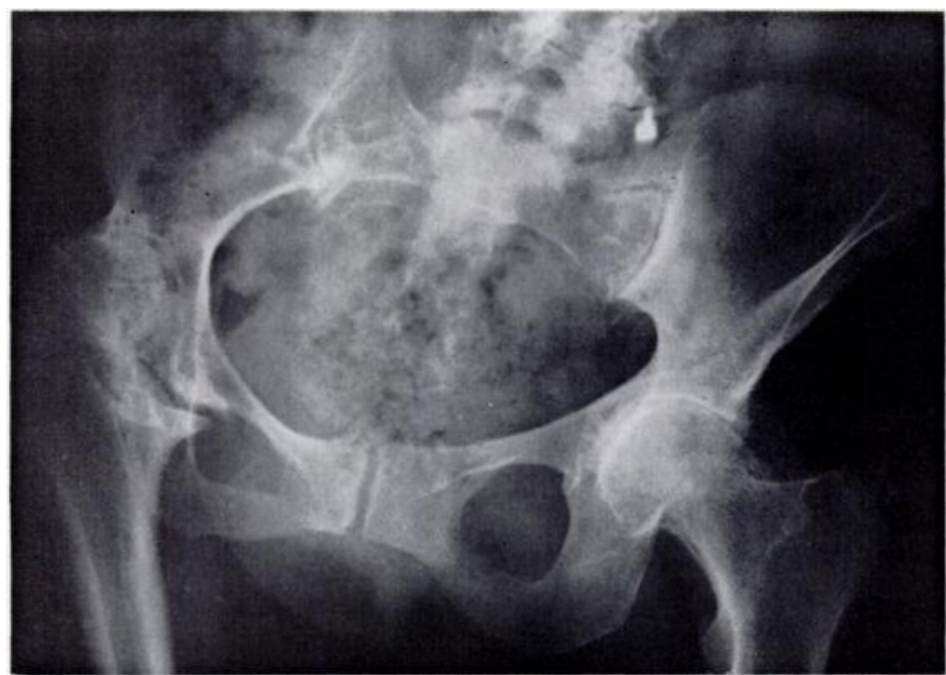

Fig.1

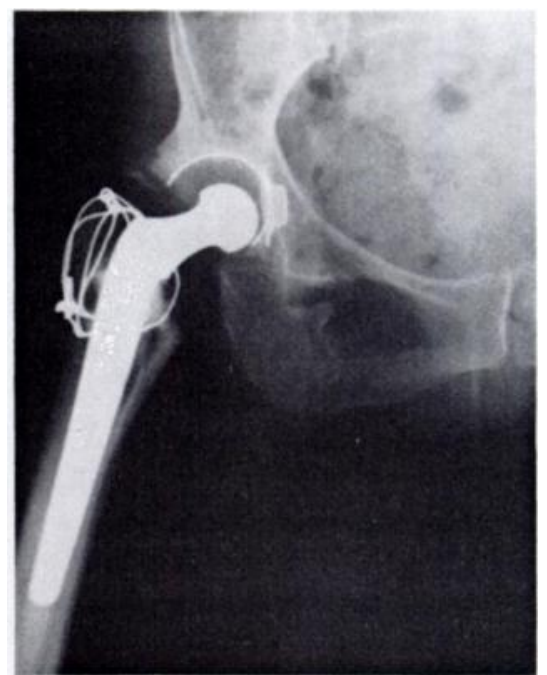

Fig. 2

Figure 1-The pelvis of a sixty-seven-year-old woman who had had a tuberculous right hip from the age of two to five years but had regained good function in adolescence to middle age, fulfilling a distinguished career in amateur tennis, until she experienced increasing pain over the last ten years. Figure 2-Vast improvement with low-friction arthroplasty. Note the offset bone cup, used because of hypoplastic pelvis.

K. Hardinge, M.Ch.Orth., F.R.C.S., Consultant Orthopaedic Surgeon $\}$ Centre for Hip Surgery, Wrightington Hospital, Appley Bridge, Sir John Charnley, C.B.E., F.R.S., F.R.C.S.

Wigan, Lancashire WN6 9EP, England.

J. Cleary, M.Ch.Orth., F.R.C.S., Department of Orthopaedic Surgery, University of Liverpool, P.O. Box 147. Liverpool L69 3BX. England

Requests for reprints should be sent to Mr K. Hardinge. 
"effective cure" of the infective arthritis and the onset of the degenerative symptoms had varied in quality and duration. The patients in their seventh decade had often enjoyed quite good function, many of them managing occupations which had involved long periods of standing (Figs. 1 and 2), whereas other patients, notably those in their fourth decade, had never enjoyed good function, having been unable to play games at school and having eschewed walking and standing whenever possible (Fig. $3)$. At the time of arthroplasty, however, all of the

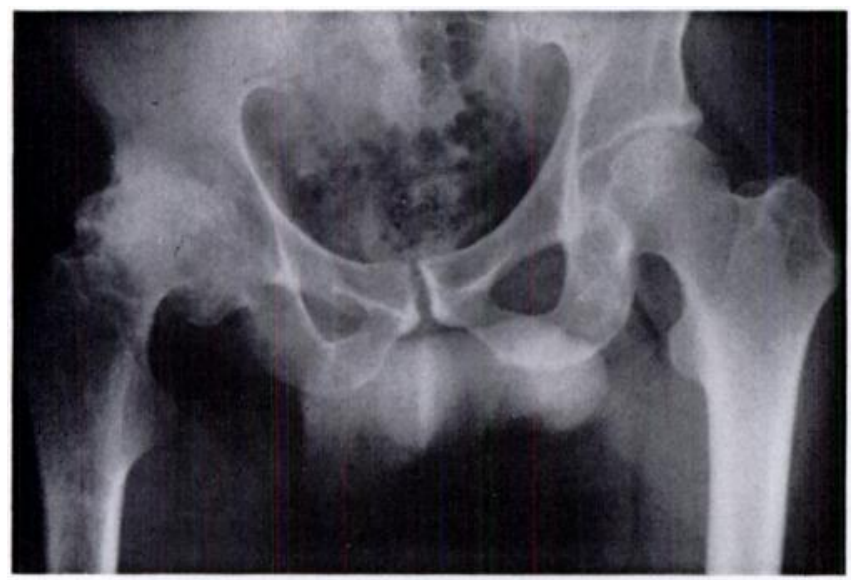

Fig. 3

The pelvis of a forty-seven-year-old man who had suffered from a tuberculous right hip from the age of seven to eleven years. He was never able to play games and obliged to take sedentary occupations because of aching and stiffness. Note the sclerosis of subarticular bone, complete loss of joint space with marginal osteophytes indicating a slowly evolving osteoarthritis.

patients passed the "pseudarthrosis test" (Charnley 1966) in which the disability is so severe that a primary pseudarthrosis would confer improvement in the clinical condition.

The entire group was carefully assessed before operation, being reviewed at six monthly or annual intervals usually over a period of twenty-four months. There was routine testing for full blood count and erythrocyte sedimentation rate, and serial radiographs of the pelvis were obtained.

Patients were considered for low-friction arthroplasty when they satisfied the following criteria: disability was sufficiently severe to pass the Charnley pseudarthrosis test; sinuses had not discharged for twenty years; quiescence of the hip infection had lasted for a definite period, the patient having enjoyed good systemic health and reasonable walking function on the "effectively cured" hip before the onset of further symptoms; deterioration had been gradual, usually noted as occurring over a period of three to five years as opposed to a period of months; the serial radiographs showed a slow progression of secondary degenerative arthritis-a gradual decreasing joint space, sclerosis of the joint margins and formation of oesteophytes; the serial blood investigations were within the normal limits over the period of observation.

Arthroplasty was performed in the routine manner using the lateral approach with reflection of the greater trochanter. Specimens from the synovium and bone marrow of the shaft of the femur were taken routinely, and the contents of any cystic cavity in the bone were considered particularly important (Fig. 4). These specimens underwent direct staining and culture for Gram-positive and tubercle bacilli. Antibiotics were not given to the patients, and the cement used for fixing the implants was standard CMW orthopaedic bone cement, which does not contain antibiotics.

\section{RESULTS}

Delay in healing occurred in five out of thirty-nine hips; in two of these, the serum oozing from the wound produced coagulase-negative Staphylococcus aureus on culture. Both patients were given appropriate antibiotics for four weeks and the wounds healed. All of the other wounds had healed in two weeks, which is normal.

There has not been any breakdown or discharge from any of these hips since the operation; the follow-up has been eight years in the longest case and ten months in the shortest case, average thirty-two months.

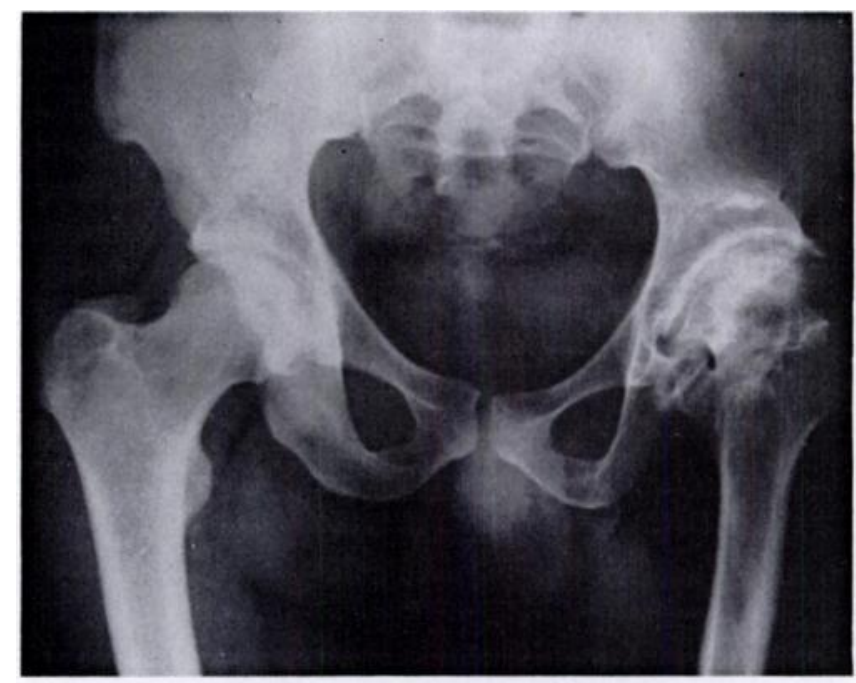

Fig. 4

The pelvis of a fifty-six-year-old man who had had a tuberculous left hip from the age of seven to ten years with sinuses over the lateral aspect. Note the large "cysts" in the neck of the femur which contained inspissated pus that failed to produce any growth on culture. Arthroplasty has remained clinically and radiologically satisfactory for four years.

The organisms cultured from the specimens of synovium, bone marrow and from the contents of bony cysts are shown in Table I. The cysts often contained old blood or serous fluid. In one case a cyst contained thickened pale-yellow pus. The arthroplasty was completed in the normal manner and the patient was given prophylactic cloxacillin for two weeks. Culture of the 
Table I. Organisms cultured from specimens of synovium, bone marrow and the contents of bone cysts

\begin{tabular}{|c|l|l|}
\hline $\begin{array}{c}\text { Case } \\
\text { number }\end{array}$ & \multicolumn{1}{|c|}{ Organism(s) } & \multicolumn{1}{c|}{$\begin{array}{c}\text { Number of } \\
\text { specimens }\end{array}$} \\
\hline 1 & $\begin{array}{l}\text { Streptococcus faecalis }+ \\
\text { coagulase-negative staphyloccus }\end{array}$ & $\begin{array}{l}\text { One specimen out } \\
\text { of three; } \\
\text { enrichment media } \\
\text { One specimen only, } \\
\text { out of three }\end{array}$ \\
7 & Aerobic spore bearer & $\begin{array}{l}\text { One specimen only, } \\
\text { out of three; } \\
\text { enrichment media } \\
\text { Three specimens } \\
\text { out of three } \\
\text { One specimen only, } \\
\text { out of four; } \\
\text { enrichment media } \\
\text { One specimen only, } \\
\text { out of four }\end{array}$ \\
\hline 26 & $\begin{array}{l}\text { Species of Klebsiella }+ \\
\text { Citrobacter freundii }\end{array}$ \\
\hline
\end{tabular}

pus failed to grow any organisms and the wound healed in the normal manner. The arthroplasty has remained clinically and radiologically satisfactory for four years.

In only one patient (Case 12) did all three samples show a positive culture, which was of coagulase-negative staphylococci. This patient was given ampicillin and cloxacillin for three months after operation and has remained clinically and radiologically satisfactory for five and a half years.

Comparison of grades before and after operation. A numerical code can provide a means of comparing the state of a joint before and after operation. The methods used in this unit follows that of Merle d'Aubigné and Postel (1954), which grades the hip by three sets of numbers from 1 to 6 , pain being judged by the first set, walking function by the second and the total range of movement by the third; the figure 6 represents normality. The addition of decimal figures indicates the predominant grading within a group. Thus if the result for a group is given as 4.75 this would indicate that 75 per cent of the group were graded 5 and the remainder 4 . In this way a fairly accurate impression of the general state of affairs can be rapidly gained.

Pain, function and movement. The average gradings before operation and at follow-up for thirty-nine hips are shown in Table II. The most impressive improvement was the relief of pain; these patients had been

Table II. Average results of numerical grading before, and after low-friction arthroplasty

\begin{tabular}{|l|c|c|}
\hline & Before operation & After operation \\
\hline Pain & 3.3 & 5.9 \\
Function & 2.9 & 5.1 \\
Movement & 2.1 & 5.0 \\
\hline
\end{tabular}

made to wait a long time before being considered for operation; and it was noteworthy that very few complained of the minor aching around the trochanter that occasionally mars what is otherwise an excellent clinical and radiological result.

\section{DISCUSSION}

An exacerbation of pain and stiffness in a previously infected joint leads to a suspicion of reactivation of the infection. This has been held to be more common in tuberculous infection with its tendency to chronicity than in acute septic infections. If the acute phase of the infection has been followed by a long period of quiescence with good function, then it is reasonable to assume that resolution may be complete. On the other

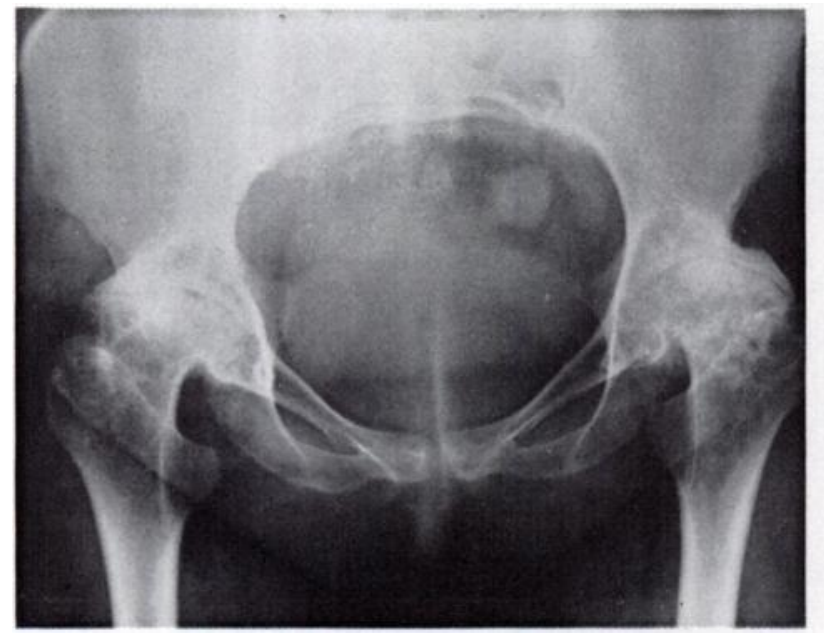

Fig. 5

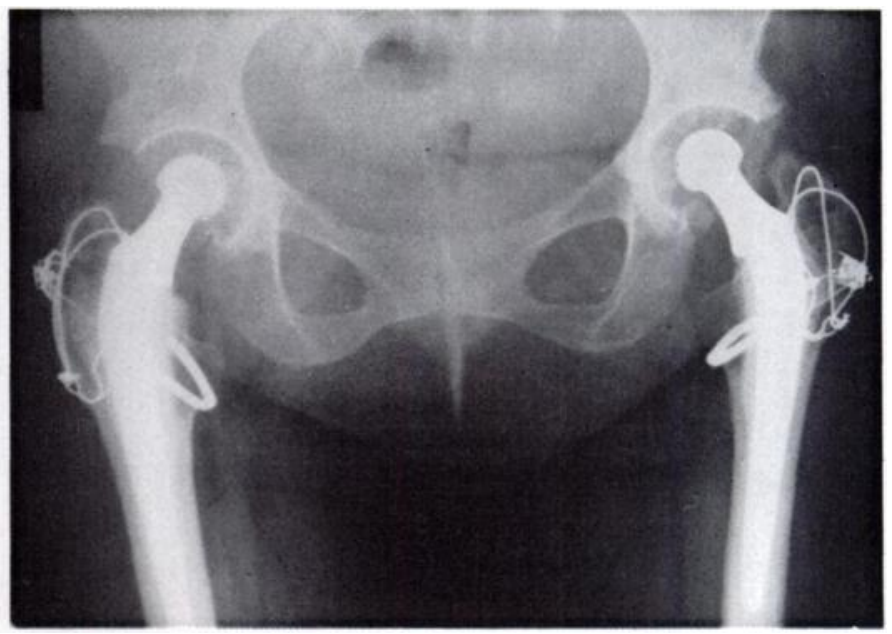

Fig. 6

Figure 5-The pelvis of a fifty-six-year-old woman showing healed septic arthritis of the hips. Note the sclerosis of subarticular bone, severe loss of joint space and marginal osteophytosis indicating slow evolution of degenerative arthritis. Radiographs of this patient changed significantly over a four-year period. Figure 6 -Three years after successful bilateral low-friction arthroplasty the numerical grading of function changed from $321: 321$ to $665: 665$. 
hand, if infection is still present within the joint one would expect it to be possible to produce cultures of the causal organism. In only one patient (Case 12) were organisms obtained from all three specimens taken at operation. and the coagulase-negative staphylococci that were cultured are not usually incriminated in primary bone infection. The occasional positive cultures seen in the other cases could be taken, in conjunction with acceptable clinical findings, to be insignificant and might represent artefactual contamination (Percival 1978).

The secondary osteoarthritis supervening in resolved infection is noteworthy for a slow progression over many years and for characteristic radiological features (Figs. 5 and 6 ). If reactivation of a primary infection does take place, there is a rapid progression in symptoms and distinct radiographic appearances. Figure 7 is the radiograph of a man aged fifty-seven who had had a tuberculous left hip from the age of seven to eleven and had until the age of fifty-six enjoyed excellent function, working as a ship's rigger. He then experienced rapid onset, over nine months, of pain and stiffness of the hip and was found on examination to have muscle spasm around the hip. The radiographs showed a wide joint space and porosis of the bone ends; biopsy of the hip showed exuberant florid synovium. These clinical appearances taken in conjunction with the rapid

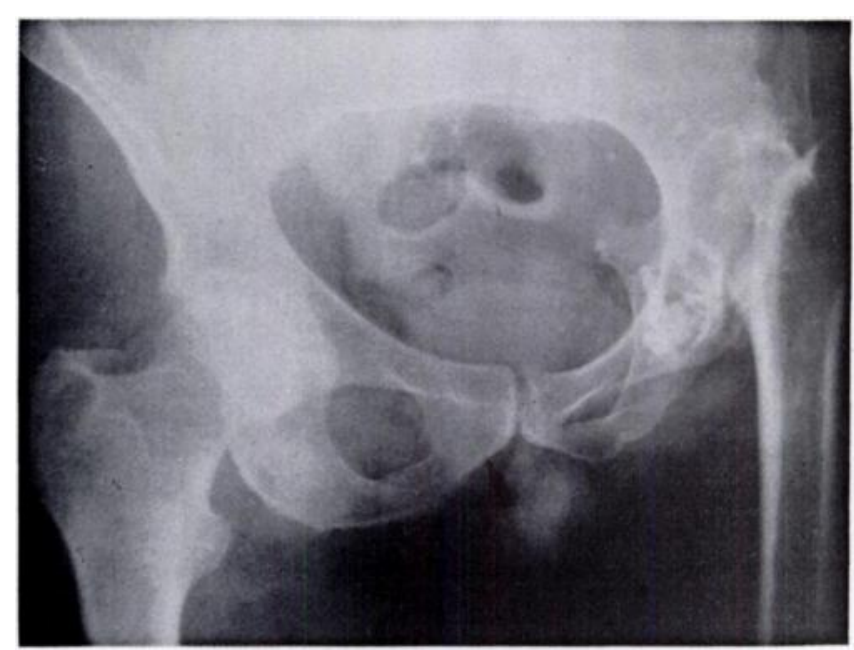

Fig. 7

The pelvis of a fifty-seven-year-old man with a history of a tuberculous left hip from the age of seven to eleven years, excellent function until the age of fifty-six years, then nine months of rapid clinical deterioration with spasm of the hip. Note the widened joint space and porosis of the femur and acetabulum. Histological examination of the synovium confirmed active tuberculosis. Low-friction arthroplasty was never contemplated in this case because of the rapidity of deterioration and the radiographic changes.

deterioration and radiographic appearances militated against total hip replacement. Histological examination confirmed active tuberculosis and the patient is now undergoing specific treatment for his reactivation.

We thank Mr R. W. E. Knowles for permission to study his patient.

\section{REFERENCES}

Charnley, J. (1966) Société International de Chirurgie Orthopédique et de Traumatologie. Tenth ('ongress. Paris /966. Paris: Masson et Cic. Merle d'Aubigné, R., and Postel, M. (1954) Functional results of hip arthroplasty with acrylic prosthesis. Journal of Bone and Joint Surgery. 36-A, $4.51-476$.

Percival, A. (1978) Consultant Microbiologist. Liverpool Royal Infirmary. Personal communication. 